\title{
Density of states and extent of wave function: two crucial factors for small polaron hopping conductivity in $1 \mathrm{D}$
}

\author{
Margarita Dimakogianni, Constantinos Simserides, and \\ Georgios P. Triberis \\ University of Athens, Physics Department, Panepistimiopolis, 15784 Zografos, \\ Athens, Greece \\ E-mail: mdimak@phys.uoa.gr, csimseri@phys.uoa.gr, gtriber@phys.uoa.gr
}

\begin{abstract}
We introduce a theoretical model to scrutinize the conductivity of small polarons in one-dimensional disordered systems, focusing on two crucial -as will be demonstrated-factors: the density of states and the spatial extent of the electronic wave function. The investigation is performed for any temperature up to $300 \mathrm{~K}$ and under electric field of arbitrary strength up to the polaron dissociation limit. To accomplish this task we combine analytical work with numerical calculations.
\end{abstract}

PACS numbers: 71.38.-k, 72.80.-r, 72.80.Ng, 73.63.-b, 71.20.-b, 73.20.At 


\section{Introduction}

The density of states (DOS) is the heart of any physical system in the sense that its structure and magnitude crucially affect all physical properties. Particularly, this holds for the response of charge carriers to external stimuli such as electric or magnetic fields, temperature gradients or temperature variations, i.e. the transport properties. In disordered materials, the random distribution of their constituents drastically affects the character of the carriers and the transport mechanisms. Under certain circumstances, the presence of disorder induces carrier localization and hopping becomes the chief transport mechanism. Hence, the electronic wave function spatial extent $\left(\alpha^{-1}\right)$, being a measure of the carrier localization, becomes a parameter of vital importance.

One-dimensional (1D) systems have been recently considered to be among the most promising materials for nanotechnology. In particular, an increasing amount of experimental and theoretical work has been devoted to the electrical properties of 1D amorphous semiconductors, amorphous carbon, doped polymers, conjugated polymers and organic materials [1, 2, 3, 4, 5, 6, 17, 8, 9, 10, 11], [12, and references therein].

Given that DNA has been placed among the most promising organic materials for nanotechnology, Triberis et al. [13], studied DNA as a 1D disordered molecular "wire" in which small polarons are the charge carriers. Based on the Generalized Molecular Crystal Model (GMCM) [14] and theoretical percolation arguments, they studied small polaron hopping along the DNA double helix and in the presence of low electric field $(F)$. Ignoring the effect of correlations, an analytical expression for the strong temperature $(T)$ dependence of the electrical conductivity $(\sigma)$ was obtained which reproduced the experimental data reported for $\lambda$-DNA [15] and for poly(dA)poly(dT) DNA [16] at high temperatures. The theoretical analysis also permitted the evaluation of the maximum hopping distance and its $T$-dependence, supporting the idea of multi-phonon assisted hopping of small polarons between next nearest neighbors of the DNA molecular "wire". Taking into account the effect of correlations $(c r)$, Triberis and Dimakogianni [12, 17] showed that $\ln \sigma^{c r} \propto T^{-1 / 2}$ holds for high as well as for low temperatures. This reproduced the strong $\sigma(T)$ at high temperatures reported for $\lambda$-DNA [15, 18] and poly(dA)-poly(dT) DNA [16], while, including correlations, the evaluation of the maximum hopping distance led to systematically longer values than those evaluated ignoring correlations [13], supporting experimental evidence for long range charge migration along the DNA double helix [19, 20, 21].

In addition, even under moderate electric fields, strong nonlinearities of $\sigma(F)$ in $1 \mathrm{D}$ disordered systems have been observed. In the variable range hopping regime and at low temperatures, Fogler and Kelley [22] investigated theoretically the effect of a finite electric field on the resistivity. They took into account the existence of highly resistive segments (breaks) on the conducting path of the carriers in 1D systems and found that the role of the breaks diminishes and eventually becomes insignificant as $F$ increases. Ma et al. 23] described hopping transport and the conductivity of 1D systems with off-diagonal disorder. Investigating the $T$-dependence of the hopping conductivity, 
they showed that it increases with the increase of $T$ taking much larger values than in the case of the Anderson model with pure diagonal disorder. They also studied the $F$ dependence of the conductivity to find that at low $F$ the hopping conductivity conforms with the ohmic law, but at strong fields it presents non-ohmic characteristics.

Triberis and Dimakogianni [24] studied the $\sigma(T, F)$ behaviour under the influence of moderate electric fields up to $\sim 10^{5} \mathrm{Vm}^{-1}$, when small polarons are transported in a disordered 1D environment, at high and low temperatures. The analytical expressions obtained for $\sigma(F, T)$, were applied to experimental findings concerning charge transport in polydiacetylene quasi-1D single crystals [9]. It was shown that at low electric fields the hopping conductivity conforms with the ohmic law while increasing the electric field the conductivity presents non-ohmic characteristics. The transition from the ohmic to the non-ohmic behaviour starts for smaller values of $F$ at lower temperatures and the rate of the increase of $\sigma$ is greater the lower $T$ is. These conclusions were in a qualitative agreement with theoretical results referred to variable range hopping [22, 23, 25]. Dimakogianni and Triberis [26] also investigated the effect of correlations on the non-ohmic behaviour of the small polaron hopping conductivity in 1D and 3D disordered systems. They concluded that the inclusion of correlations results to a much stronger dependence of the conductivity on the magnitude of the applied electric field compared to the uncorrelated case. The deviation of the conductivity from the ohmic behaviour appears twice as fast when correlation effects are taken into account, for a given applied electric field as the temperature increases.

In the present work, taking into account the directionality imposed by the electric field on the transport path of the carriers, we examine the role of the magnitude of the density of states and the extent of the electronic wave function and calculate $\sigma$. The aim of the present work, is to investigate $\sigma(T, F)$ for all reasonable $T$ and $F$ values, i.e. from 10 up to $300 \mathrm{~K}$ and up to the $F$ values where polarons cease to exist. This is done varying the density of states by orders of magnitude around values which are relevant to common 1D systems [27, 28, 29, 30] and varying the extent of the electronic wave function from 1 to $5 \AA$, i.e. reasonable values for common organic molecules [31, 32]. We demonstrate that $F$ plays both a constructive energetic role by offering energy for the carrier jumps and simultaneously a destructive role, in the sense that the stronger it is the more it forces the polaron to jump opposite to the $\boldsymbol{F}$ direction prohibiting forward jumps to neighboring sites.

In Section 2 we present our theoretical model including the basic analytical expressions at high and low temperatures. According to the mathematical analysis of the Generalized Molecular Crystal Model [14, 33], it is the condition $\hbar \omega_{0} \ll k_{B} T$ $\left(\hbar \omega_{0} \gg k_{B} T\right)$ that determines the high (low) temperature regime. This mathematical analysis leads to the evaluation of the intrinsic transition rate, which differs at high temperatures (multi-phonon assisted hopping), compared to that at low temperatures (few-phonon assisted hopping). Which temperature range in real systems is indeed high or low depends on the system under study. Our numerical results together with the relevant discussion are staged in Section 3, In this way we examine the conductivity of 
small polarons in one-dimensional disordered systems, and demonstrate that the density of states and the spatial extent of the electronic wave function are two crucial factors for its behaviour. The temperature and the electric field ranges that we consider are very broad. In particular, the electric field is varied from very low up to the polaron dissociation limit $\left(\sim 1 \times 10^{8} \mathrm{Vm}^{-1}\right)$. Finally, in Section 4 we state our conclusions.

\section{Theory}

\subsection{Generalized Molecular Crystal Model}

In the context of GMCM we consider a 1D deformable "wire" consisting of "molecular lattice sites" across which small polarons are transported in the presence of disorder. By $\epsilon_{i}(0)$, and $\epsilon_{j}(0)$ we denote the energies of an electron on site at vector positions $\mathbf{r}_{i}$ and $\mathbf{r}_{j}$, respectively, if the "molecular lattice sites" are constrained not to be displaced in response to the presence of the electron. Due to the disorder these local electronic energies, $\epsilon_{i}(0)$, and $\epsilon_{j}(0)$ are not equal. The energetic non-equivalence of the two sites will affect the small polaron's binding energy, $E_{b}(i)$, in the sense that, the lower the local electronic energy is the more localized the electronic wave function will tend to be and consequently the larger its binding energy will be. Assuming that the stiffness of the "molecular lattice" is unaltered, the difference in binding energy means a difference in the electron-lattice interaction parameters $A_{i}$ and $A_{j}$ i.e. $E_{i}\left(\mathbf{x}_{i}\right)=\epsilon_{i}(0)-A_{i} \mathbf{x}_{i}$ and $E_{j}\left(\mathbf{x}_{j}\right)=\epsilon_{j}(0)-A_{j} \mathbf{x}_{j}$ with $A_{i} \neq A_{j}$. Here, $E_{i}\left(\mathbf{x}_{i}\right)$ is the electronic energy of the system of the electron and the isolated molecule with configurational coordinate $\mathbf{x}_{i}$, which represents the deviation of the atoms of the molecule at position $\mathbf{r}_{i}$ from their equilibrium configuration i.e. the local vibrational displacement coordinate.

The GMCM [14] is based on a generalized "hopping model" Hamiltonian of the form

$$
<m|H| n>=<m\left|H_{0}+V\right| n>=E_{i,\left\{n_{\mathbf{k}}\right\}} \delta_{i j} \delta_{\left\{n_{\mathbf{k}}\right\},\left\{n_{\mathbf{k}^{\prime}}\right\}}+<m|V| n>,
$$

the $<m|V| n>$ term [14] is the overlap part of the Hamiltonian, $|n>=| i,\left\{n_{\mathbf{k}}\right\}>$ are the eigenstates of $H$, and $H_{0}$ is the zeroth-order (i.e. for electronic overlap integral of the tight-binding theory $J=0$ ) Hamiltonian with corresponding eigenvalues

$$
E_{i,\left\{n_{\mathbf{k}}\right\}}=\epsilon_{i}(0)-E_{b}(i)+\sum_{\mathbf{k}} \hbar \omega_{\mathbf{k}}\left(n_{\mathbf{k}}+\frac{1}{2}\right) .
$$

Here, $\left\{n_{\mathbf{k}}\right\}$ represents the totality of the vibrational quantum numbers $\left(\ldots, n_{\mathbf{k}}, \ldots\right)$ for the occupation of the site with position vector $\mathbf{r}_{i}$, and

$$
E_{b}(i)=\frac{1}{N} \sum_{\mathbf{k}}\left(A_{i}^{2} / 2 M \omega_{\mathbf{k}}^{2}\right),
$$

is the small polaron binding energy. $N$ is the number of "molecular lattice sites" and $M$ is the appropriate reduced atomic mass. The relation between $\omega_{\mathbf{k}}$ and its associated wavevector $\mathbf{k}$, i.e. the dispersion relation, is given by:

$$
\omega_{\mathbf{k}}^{2}=\omega_{0}^{2}+\omega_{1}^{2} \sum_{\mathbf{k}} \cos \left(\mathbf{k} \cdot \mathbf{h}^{\prime}\right)
$$


where $|\mathbf{k}|=2 \pi p / N$, the integer $p$ lying in the range $-(N-1) / 2 \leq p \leq(N-1) / 2$, and $\mathbf{h}^{\prime}$ indexes the nearest neighbors $\left(\mathbf{r}_{i}+\mathbf{h}^{\prime}\right)$ of an arbitrary site $\mathbf{r}_{i}$. $\omega_{0}$ is the harmonic oscillator frequency associated with the configurational coordinate of the isolated molecule. The relation $\omega_{1} \ll \omega_{0}$ determines the weak dispersion limit.

Equations (2) and (3) show the essential features of the GMCM which are:

1. site-dependent local electronic energy $\epsilon_{i}(0)$.

2. site-dependent electron-lattice interaction parameter, $A_{i}$, and concomitant binding energy, $E_{b}(i)$.

The knowledge of $\langle m|V| n>$, permits the evaluation of the "microscopic" small polaron velocity operator [34, 35],

$$
\mathbf{u}_{i j}=<m|\mathbf{u}| n>=\left(\frac{i}{\hbar}\right)<m|V| n>\left(\mathbf{r}_{j}-\mathbf{r}_{i}\right),
$$

the charge current density operator,

$$
\mathbf{j}_{i j}=n_{c} q \mathbf{u}_{i j},
$$

where $n_{c}$ is the charge carrier concentration, and $q$ is the carrier's charge, and thus the "microscopic" electrical conductivity [36],

$$
\sigma_{i j}=\int_{0}^{\infty} d t \int_{0}^{\beta} d \rho<\mathbf{j}(-i \hbar \rho) \mathbf{j}(t)>,
$$

where $\beta=1 / k_{B} T$. The mobility, $\mu_{i j}$, and consequently the diffusion constant, given by $D_{i j}=\mu_{i j} / e \beta$, are determined and lead to the "microscopic" jump rate which reads:

$$
L_{i j}=\frac{D_{i j}}{\left|\mathbf{r}_{i}-\mathbf{r}_{j}\right|^{2}}
$$

Assuming that the dependence on the spatial separation $R_{i j}$, of the two sites is [37] $\exp \left(-2 \alpha R_{i j}\right)$, the "microscopic" intrinsic transition rate, $\gamma_{i j}$, for a small polaron hopping from a site $i$ to an empty site $j$ is given by

$$
\gamma_{i j}=\exp \left(-2 \alpha R_{i j}\right) L_{i j}
$$

The treatment refers to the non(anti)-adiabatic limit, i.e. in the physical situation where the electron is no longer able to follow rapid fluctuations of the lattice and, hence, it does not respond quickly enough to the occurrence of a coincident event in order to overcome the energy barrier. In this case, $J$ can be treated as a small perturbation in the lowest order [34, 38, 39].

The expansion of the model to include the influence of possible strong local interparticle correlations might be interesting as intercarrier interactions exist in real systems. However, this is beyond the aim of the present work.

\subsection{Hopping at high temperatures}

When a carrier hops from site $i$ of energy $E_{i}$ to site $j$ of energy $E_{j}$, at a distance $R_{i j}$, the intrinsic transition rate between the two localized states at high ( $h$ ) temperatures $\left(\hbar \omega_{0} \ll k_{B} T\right.$ [14] $)$ is

$$
\gamma_{i j}^{h}=\gamma_{0}^{h} \exp \left(-2 \alpha R_{i j}\right) \exp \left(-\frac{\varepsilon_{2}}{k_{B} T}\right) \times \begin{cases}\exp \left(-\frac{E_{j}-E_{i}}{2 k_{B} T}\right), & E_{j}>E_{i} \\ \exp \left(\frac{E_{i}-E_{j}}{2 k_{B} T}\right), & E_{j}<E_{i}\end{cases}
$$



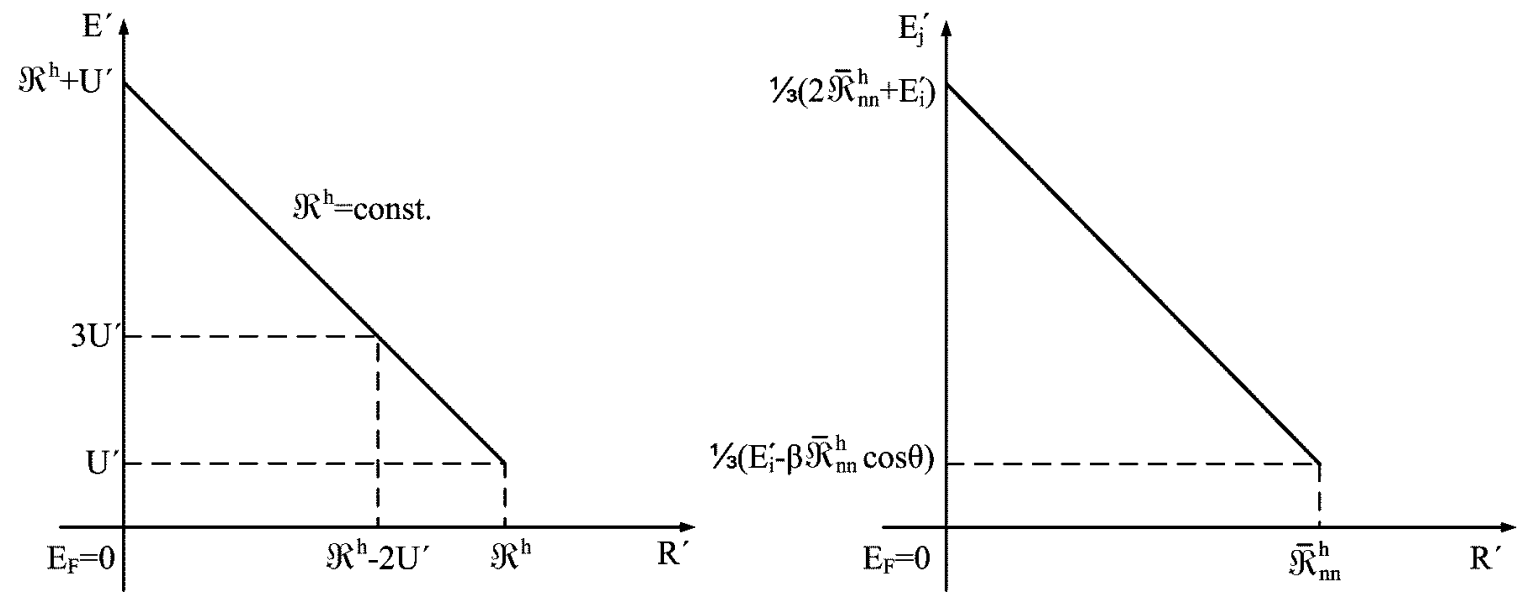

Figure 1. High temperatures. (I) Left panel. Contour of constant $\Re^{h}$ for an initial site of $U^{\prime}$. All possible final sites for the carrier, $\mathcal{N}\left(\Re^{h}\right)$, lie on or within the contour $\Re^{h}$, for a particular $\theta$. (II) Right panel. Contour of integration $\Re^{h}=\bar{\Re}_{n n}^{h}$, for an initial site of $E_{i}^{\prime}$ and particular $\theta$, for the evaluation of ${\overline{R^{\prime}}}_{F}^{h}$.

Here $\alpha^{-1}$ is the spatial extent of the electronic wave function, $\gamma_{0}^{h}=\left(J^{2} / \hbar\right)\left(\pi / 4 \varepsilon_{2} k_{B} T\right)^{1 / 2}$ and $\varepsilon_{2}=\left[E_{b}(i)+E_{b}(j)\right] / 4 . E_{b}(i)$ and $E_{b}(j)$ is the small polaron binding energy for sites $i$ and $j$, respectively. Hence, $\gamma_{i j}^{h}$ (as well as $\gamma_{i j}^{l}$ ) have both spatial and energy dependence [14, 33]. The spatial dimensions of the system and the number of energies involved in the expression of the intrinsic transition rate can be considered as the coordinates of a "hopping space" in which the small polaron transport occurs under the influence of $F$. In this "hopping space", the most probable hop for a carrier on a site at energy $E_{i}$ is to the empty site at closest range, i.e. to its nearest neighbor site. The average nearest neighbor range in the "hopping space", $\bar{R}_{n n}$, determines the conductivity of the system [40]. Thus, to evaluate the electrical conductivity we have to calculate this quantity first. Then, taking into account that in real space greater real forward distances will be hopped in the downfield direction rather than upfield, an average real forward distance hopped should be evaluated which, as will be presented in the following (cf. Eqs. 33. 34), leads to the mobility of the carriers and finally the overall conductivity of the system.

From the expression of the intrinsic transition rate between two sites $i$ and $j$, we define the range $\Re_{i j}^{h}$ between the sites in the "hopping space"

$$
\Re_{i j}^{h}=2 \alpha R_{i j}+\left(\frac{\varepsilon_{2}}{k_{B} T}\right)+ \begin{cases}\frac{E_{j}-E_{i}}{2 k_{B} T}, & E_{j}>E_{i} \\ -\frac{E_{i}-E_{j}}{2 k_{B} T}, & E_{j}<E_{i}\end{cases}
$$

Taking the energies of the carrier to be mainly polaronic [14], and using for convenience the terms $E_{i}$ and $E_{j}$ instead of $E_{b}(i)$ and $E_{b}(j)$ respectively, we obtain $\varepsilon_{2}=\left(E_{i}+E_{j}\right) / 4$. Therefore

$$
\Re_{i j}^{h}=2 \alpha R_{i j}+\frac{3 E_{j}-E_{i}}{4 k_{B} T}
$$


where $E_{j}>E_{i}$ for absorption and $E_{i} / 3<E_{j}<E_{i}$ for emission of phonons. Introducing the dimensionless coordinates $R_{i j}^{\prime}=2 \alpha R_{i j}, E_{i}^{\prime}=E_{i} / 2 k_{B} T$ and $E_{j}^{\prime}=E_{j} / 2 k_{B} T$,

$$
\Re_{i j}^{h}=R_{i j}^{\prime}+\frac{3}{2} E_{j}^{\prime}-\frac{1}{2} E_{i}^{\prime},
$$

where $E_{j}^{\prime}>E_{i}^{\prime}$ for absorption and $E_{i}^{\prime} / 3<E_{j}^{\prime}<E_{i}^{\prime}$ for emission.

Under the influence of an externally applied electric field the actual energy of the hop is modified [40]

$$
\frac{3}{2} E_{j}^{\prime}-\frac{1}{2} E_{i}^{\prime} \longrightarrow \frac{3}{2} E_{j}^{\prime}-\frac{1}{2} E_{i}^{\prime}+\frac{\beta}{2} R_{i j}^{\prime} \cos \theta,
$$

where $\beta=e F / 2 \alpha k_{B} T$ and $\theta$ is the angle between the directions of $R_{i j}^{\prime}$ and $F$. Defining the reduced initial $\left(U^{\prime}\right)$ and final $\left(E^{\prime}\right)$ coordinates in the "hopping space"

$$
U^{\prime}=\frac{1}{2} E_{i}^{\prime}, \quad E^{\prime}=\frac{3}{2} E_{j}^{\prime}+\frac{\beta}{2} R_{i j}^{\prime} \cos \theta,
$$

the range between two sites in the "hopping space" becomes

$$
\Re^{h}=R^{\prime}+E^{\prime}-U^{\prime},
$$

where $E^{\prime}>3 U^{\prime}$ for absorption and $U^{\prime}<E^{\prime}<3 U^{\prime}$ for emission. The indices from $\Re_{i j}^{h}$ and $R_{i j}^{\prime}$ have been dropped.

For the evaluation of the average nearest neighbor range, $\bar{\Re}_{n n}^{h}$, firstly we have to evaluate the number of unoccupied sites within a range $\Re^{h}$ of a particular site of $U^{\prime}$ as a function of $T$ and $F, \mathcal{N}\left(\Re^{h}\right)$. The three-dimensional "hopping space" can be represented, for a particular $\theta$ by a two-dimensional diagram (Fig. 1 (I)).

For hops of range less or equal to $\Re^{h}$ from an initial site of $U^{\prime}$, the final sites will lie on or within the contour $\Re^{h}$, for a particular $\theta$, i.e. in the space defined by $U^{\prime}<E^{\prime}<\left(\Re^{h}+U^{\prime}\right)-R^{\prime}$ and $0<R^{\prime}<\Re^{h}$ (Fig. 1(I)). Thus, using Eqs. 15, the number of empty sites enclosed by the contour $\Re^{h}$ is

$$
\mathcal{N}\left(\Re^{h}\right)=\sum_{0, \pi} \int_{0}^{\Re^{h}} \int_{\mathcal{L L}}^{\mathcal{U L}} N\left(E_{j}^{\prime}\right)\left[1-f\left(E_{j}^{\prime}\right)\right] \frac{k_{B} T}{\alpha} d E_{j}^{\prime} d R^{\prime} .
$$

$\mathcal{L} \mathcal{L}=\frac{1}{3}\left(E_{i}^{\prime}-\beta R^{\prime} \cos \theta\right), \mathcal{U L}=\frac{1}{3}\left[2 \Re^{h}+E_{i}^{\prime}-2 R^{\prime}\left(1+\frac{\beta}{2} \cos \theta\right)\right]$ and $\theta=0, \pi$ as we have chosen to put $F$ exactly along the $1 \mathrm{D}$ axis where transport takes place. $N\left(E_{j}^{\prime}\right)$ is the density of states, and $f\left(E_{j}^{\prime}\right)$ the Fermi-Dirac distribution. We take the Fermi energy $E_{F}=0$. Assuming a constant density of states, $N\left(E_{j}^{\prime}\right)=N\left(E_{i}^{\prime}\right)=N_{0}$,

$$
\mathcal{N}\left(\Re^{h}\right)=\mathcal{C} \sum_{0, \pi} \int_{0}^{\Re^{h}} \ln \frac{1+e^{\frac{2}{3}\left(2 \Re^{h}+E_{i}^{\prime}-2 R^{\prime}\left(1+\frac{\beta}{2} \cos \theta\right)\right)}}{1+e^{\frac{2}{3}\left(E_{i}^{\prime}-\beta R^{\prime} \cos \theta\right)}} d R^{\prime},
$$

where $\mathcal{C}=\frac{N_{0} k_{B} T}{2 \alpha}$. 

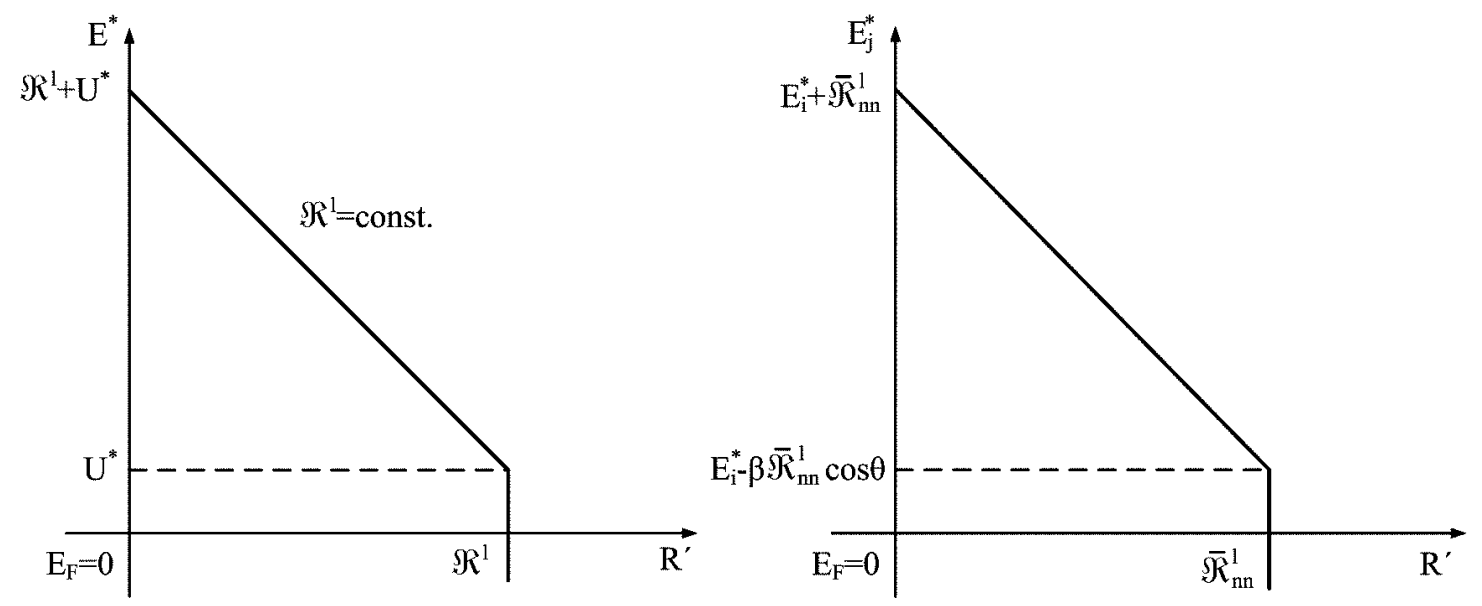

Figure 2. Low temperatures. (I) Left panel. Contour of constant $\Re^{l}$ for an initial site of $U^{\prime}$. All possible final sites for the carrier, $\mathcal{N}\left(\Re^{l}\right)$, lie on or within the contour $\Re^{l}$, for a particular $\theta$. (II) Right panel. Contour of integration $\Re^{l}=\bar{\Re}_{n n}^{l}$, for an initial site of $E_{i}^{*}$ and particular $\theta$, for the evaluation of ${\overline{R^{\prime}}}_{F}^{l}$.

\subsection{Hopping at low temperatures}

The intrinsic transition rate at low (l) temperatures $\left(\hbar \omega_{0} \gg k_{B} T\right.$ [33]) is

$$
\gamma_{i j}^{l}=\gamma_{0}^{l} \exp \left(-2 \alpha R_{i j}\right)\left\{\begin{array}{ll}
\exp \left(-\frac{E_{j}-E_{i}}{k_{B} T}\right), & E_{j}>E_{i} \\
1, & E_{j}<E_{i}
\end{array} .\right.
$$

$\gamma_{0}^{l}=\frac{\omega_{0}}{\pi}\left[\frac{\pi J \exp \left(\frac{-2 \varepsilon_{2}}{\hbar \omega_{0}}\right)}{\hbar \omega_{0}}\right]^{2}\left[\left(4 \varepsilon_{2} / \hbar \omega_{0}\right)^{\Delta_{i j} / \hbar \omega_{0}} /\left(\Delta_{i j} / \hbar \omega_{0}\right) !\right]$. Following the same methodology as for the high temperatures, we assign every hop of the carrier to a hop in a threedimensional "hopping space" defined by one spatial and two energy coordinates.

From the expression of the intrinsic transition rate between two sites $i$ and $j$, we define the range $\Re_{i j}^{l}$ between the sites in the "hopping space"

$$
\Re_{i j}^{l}=\left\{\begin{array}{ll}
2 \alpha R_{i j}+\frac{E_{j}-E_{i}}{k_{B} T}, & E_{j}>E_{i} \\
2 \alpha R_{i j}, & E_{j}<E_{i}
\end{array} .\right.
$$

Introducing the dimensionless coordinates $R_{i j}^{\prime}=2 \alpha R_{i j}, E_{i}^{*}=E_{i} / k_{B} T$ and $E_{j}^{*}=$ $E_{j} / k_{B} T$

$$
\Re_{i j}^{l}=\left\{\begin{array}{ll}
R_{i j}^{\prime}+E_{j}^{*}-E_{i}^{*}, & E_{j}^{*}>E_{i}^{*} \\
R_{i j}^{\prime}, & E_{j}^{*}<E_{i}^{*}
\end{array} .\right.
$$

Under the influence of an externally applied electric field the actual energy of the hop is modified [40]

$$
E_{j}^{*}-E_{i}^{*} \longrightarrow E_{j}^{*}-E_{i}^{*}+\beta R_{i j}^{\prime} \cos \theta
$$

where $\beta=e F / 2 \alpha k_{B} T$. Thus,

$$
\Re^{l}=\left\{\begin{array}{ll}
R^{\prime}(1+\beta \cos \theta)+E_{j}^{*}-E_{i}^{*}, & E_{j}^{*}>E_{i}^{*}-\beta R^{\prime} \cos \theta \\
R^{\prime}, & E_{j}^{*}<E_{i}^{*}-\beta R^{\prime} \cos \theta
\end{array} .\right.
$$


The indices from $\Re_{i j}^{l}$ and $R_{i j}^{\prime}$ have been dropped. Defining the reduced initial $\left(U^{\prime}\right)$ and final $\left(E^{\prime}\right)$ coordinates in the "hopping space"

$$
U^{*}=E_{i}^{*}, \quad E^{*}=E_{j}^{*}+\beta R^{\prime} \cos \theta,
$$

the range between two sites in the "hopping space" becomes

$$
\Re^{l}=\left\{\begin{array}{ll}
R^{\prime}+E^{*}-U^{*}, & E^{*}>U^{*} \\
R^{\prime}, & E^{*}<U^{*}
\end{array} .\right.
$$

For the evaluation of the average nearest neighbor range in the "hopping space", $\bar{\Re}_{n n}^{l}$, firstly we have to evaluate the number of unoccupied sites within a range $\Re^{l}$ of a particular site of $U^{*}$ as a function of $T$ and $F, \mathcal{N}\left(\Re^{l}\right)$. The "hopping space" can be represented, for a particular $\theta$ by a two-dimensional diagram (Fig. 2(I)).

For hops of range less or equal to $\Re^{l}$ from an initial site of $U^{*}$, the final sites will lie on or within the contour $\Re^{l}$, for a particular $\theta$, i.e. in the space defined by $-\infty<E^{*}<\left(\Re^{l}+U^{*}\right)-R^{\prime}$ and $0<R^{\prime}<\Re^{l}$ (Fig. 2 (I)). Thus, using Eqs. 24, the number of empty sites enclosed by the contour $\Re^{l}$ is

$$
\mathcal{N}\left(\Re^{l}\right)=\sum_{0, \pi} \int_{0}^{\Re^{l}} \int_{\mathcal{L L}}^{\mathcal{U L}} N\left(E_{j}^{*}\right)\left[1-f\left(E_{j}^{*}\right)\right] \frac{k_{B} T}{2 \alpha} d E_{j}^{*} d R^{\prime} .
$$

$\mathcal{L} \mathcal{L}=-\infty, \mathcal{U L}=\Re^{l}+E_{i}^{*}-R^{\prime}(1+\beta \cos \theta)$. Assuming a constant density of states, $N\left(E_{j}^{*}\right)=N\left(E_{i}^{*}\right)=N_{0}$,

$$
\mathcal{N}\left(\Re^{l}\right)=\mathcal{C} \sum_{0, \pi} \int_{0}^{\Re^{l}} \ln \left[1+\exp \left(\Re^{l}+E_{i}^{*}-R^{\prime}(1+\beta \cos \theta)\right)\right] d R^{\prime},
$$

where again $\mathcal{C}=\frac{N_{0} k_{B} T}{2 \alpha}$.

\subsection{Conductivity}

We define $E_{i}^{\prime \prime}=E_{i}^{\prime}$ for high temperatures or $E_{i}^{\prime \prime}=E_{i}^{*}$ for low temperatures. The knowledge of the number of unoccupied sites within a range $\Re^{h / l}$ for either high or low $(h / l)$ temperatures, $\mathcal{N}\left(\Re^{h / l}\right)$, permits the evaluation of the average nearest neighbor range, $\bar{\Re}_{n n}^{h / l}$, when the carrier resides on a particular site of $E_{i}^{\prime \prime}$, as a function of $T$ and $F[40]$

$$
\bar{\Re}_{n n}^{h / l}=\int_{0}^{\infty} \Re^{h / l} \frac{\partial \mathcal{N}\left(\Re^{h / l}\right)}{\partial \Re^{h / l}} \exp \left[-\mathcal{N}\left(\Re^{h / l}\right)\right] d \Re^{h / l},
$$

or equivalently

$$
\bar{\Re}_{n n}^{h / l}=\int_{0}^{\infty} \exp \left[-\mathcal{N}\left(\Re^{h / l}\right)\right] d \Re^{h / l} .
$$

The evaluation of $\bar{\Re}_{n n}^{h / l}$, gives the range in the three-dimensional "hopping space" where a nearest neighbor exists that can host the carrier when the carrier hops from an initial site of $E_{i}^{\prime \prime}$. However, it gives no information on the direction of the hop of the carrier.

Considering, all sites of initial $E_{i}^{\prime \prime}$ and assuming that all hops from these sites are all hops of range $\bar{\Re}_{n n}^{h / l}$, then in real space, these hops will be in random directions, but 
for a hop to final sites of the same energy, greater real forward distance will be hopped in the downfield direction rather than upfield. Thus, summing over all final sites, for initial sites of $E_{i}^{\prime \prime}$, there will be associated an average real forward distance hopped [40]

$$
\bar{R}_{F}^{h / l}=\frac{{\overline{R_{F}^{\prime}}}_{F}^{h / l}}{2 \alpha} .
$$

For high temperatures, the distance $\bar{R}_{F}^{h}$ is evaluated by averaging $R^{\prime} \cos \theta$ over the contour $\bar{\Re}_{n n}^{h}=$ const. (Fig. 11(II)), and hence

$$
{\overline{R^{\prime}}}_{F}^{h}=\frac{I_{1}}{I_{2}} .
$$

The integrals $I_{1}$ and $I_{2}$ are given in the Appendix.

For low temperatures, the distance $\bar{R}_{F}^{l}$ is evaluated by averaging $R^{\prime} \cos \theta$ over the contour $\bar{\Re}_{n n}^{l}=$ const. (Fig. 2 (II)), and hence

$$
{\overline{R^{\prime}}}_{F}^{l}=\frac{I_{1}+I_{2}}{I_{3}+I_{4}} \text {. }
$$

The integrals $I_{1}, I_{2}, I_{3}, I_{4}$ are given in the Appendix.

Either for high or for low temperatures, having calculated the distance ${\overline{R^{\prime}}}_{F}^{h / l}$ and considering that the probability of all hops is $\exp \left(-\bar{\Re}_{n n}^{h / l}\right)$, the average rate of transport of carriers is $\nu_{p h}{\overline{R_{F}^{\prime}}}_{F}^{h / l} \exp \left(-\bar{\Re}_{n n}^{h / l}\right)$. Here, $\nu_{p h}$ is a hopping attack frequency of the order of a phonon frequency, assumed the same for all hops.

The mobility for small polarons of $E_{i}^{\prime \prime}=E_{i}^{\prime}$ for high temperatures or of $E_{i}^{\prime \prime}=E_{i}^{*}$ for low temperatures reads

$$
\mu\left(E_{i}^{\prime \prime}\right)=-\frac{\nu_{p h}}{F} \frac{{\overline{R_{F}^{\prime}}}_{F}^{h / l}}{2 \alpha} \exp \left(-\bar{\Re}_{n n}^{h / l}\right),
$$

and the conductivity of the system is

$$
\sigma^{h / l}(F, T)=-\int_{-\infty}^{\infty} e N\left(E_{i}^{\prime \prime}\right) f\left(E_{i}^{\prime \prime}\right) \mu\left(E_{i}^{\prime \prime}\right) k_{B} T d E_{i}^{\prime \prime} .
$$

\section{Results and discussion}

In the following, based on the theoretical analysis presented above, we calculate numerically the electrical conductivity varying the density of states and the spatial extent of the localized electronic wave function. Our numerical results refer for simplicity reasons to a constant density of states, although, typically, in 1D systems the density of states has a strong energy dependence. One could alternatively use an energy dependent model for the density of states [41, 42] which is expected to influence somehow the conductivity. This is beyond the scope of the present paper, but could be numerically examined in the future via the same approach, as it is evident from Eq. 34 .

We consider the range $T=(160-300) \mathrm{K}$ as high temperatures and the range $T=(10-150) \mathrm{K}$ as low temperatures. We investigate the influence of an electric field in the range $F=\left(5 \times 10^{3}-1 \times 10^{8}\right) \mathrm{Vm}^{-1}$. We did not consider higher values of $F$, because 
it is generally accepted that the value of the highest electric field that the polaron can sustain is about $1 \times 10^{8} \mathrm{Vm}^{-1}$ [43, 44, 45]. We take $\nu_{p h}=10^{12} \mathrm{~s}^{-1}$.

We vary the density of states by orders of magnitude around values which are relevant to common 1D systems [27, 28, 29, 30] and the extent of the electronic wave function from 1 to $5 \AA$, i.e. for sensible values for common organic molecules [31, 32]. Specifically, in Figs. 3, 4, 5 and Figs. 8, 9, 10 we keep $\alpha^{-1}=2 \AA$, while in Figs. 6, 17 we vary $\alpha^{-1}$ in the range $1-5 \AA$. However, to keep our results as general as possible, we do not make any reference to a specific material.

\subsection{High temperatures}

For the temperature range $T=(160-300) \mathrm{K}$, Fig. 3 presents $\sigma$ as a function of $T^{-1 / 2}$ for different magnitudes of the DOS, i.e. for $N_{0}=\left(10^{9}-10^{13}\right) \mathrm{eV}^{-1} \mathrm{~m}^{-1}$ [cases (a) to (e), respectively]. $\alpha^{-1}=2 \AA . F=\left(5 \times 10^{3}-1 \times 10^{8}\right) \mathrm{Vm}^{-1}$. We depict our results as a function of $T^{-1 / 2}$ to compare them with the analytically obtained formula $\ln \sigma \propto T^{-1 / 2}$ which holds for low up to moderate electric fields and was previously obtained by two of us [26] following a different theoretical treatment and taking into account the effect of correlations, namely

$$
\sigma^{h, c r}(F, T) \propto \exp \left[-\left(\frac{T_{0}^{h, c r}}{T}\right)^{1 / 2}\left(1-\frac{F^{2}}{g(T)}\right)^{1 / 2}\right] .
$$

Here $g(T)=\left(2 \alpha k_{B} T / e\right)^{2}$ and $T_{0}^{h, c r}=1.18 \alpha / k_{B} N_{0}$. We observe that higher density of states leads to higher conductivity in such a way that $\frac{d \sigma}{d N_{0}}$ is smaller for higher densities of states. As shown in Fig. 3, in this specific example, augmenting DOS by four orders of magnitude, the conductivity rises by approximately eleven orders of magnitude.

From these results we realize that the $T^{-1 / 2}$-behaviour of $\sigma$ [26] holds for low up to moderate electric fields. For the smaller densities of states, i.e. for $N_{0}=10^{9}$ and $10^{10} \mathrm{eV}^{-1} \mathrm{~m}^{-1}, \frac{d \sigma}{d F}<0$ i.e. the conductivity is larger for lower electric field strengths. This is due to the competitive role of the directionality imposed by the electric field and the temperature. This directionality affects destructively $\sigma$ when not many sites are available for the carrier i.e. for small densities of states. Here we notice that according to Eq. 16 and Fig. 1(I), the electric field affects the range between two sites in the "hopping space" both for the absorption and the emission branch. On the contrary, this effect does not appear at low temperatures $(T=(10-150) \mathrm{K})$, discussed later on Subsection 3.2 (Fig. 8) because in the corresponding expression for the range between two sites in the "hopping space" at low temperatures (Eq. 25 and Fig. 2(I)), the electric field affects only the finite area of the absorption branch. We mention that the electric field plays a constructive role, too, due to its energy offer to the carriers. At $N_{0}=10^{11}$ $\mathrm{eV}^{-1} \mathrm{~m}^{-1}$ it seems that the available sites are numerous enough so that the directionality of the electric field hardly affects the conductivity. For higher densities of states, i.e. for $N_{0}=10^{12}$ and $10^{13} \mathrm{eV}^{-1} \mathrm{~m}^{-1}$, only the constructive energetic influence of the electric field appears. Now $\frac{d \sigma}{d F}>0$ i.e. the conductivity is larger for higher electric 

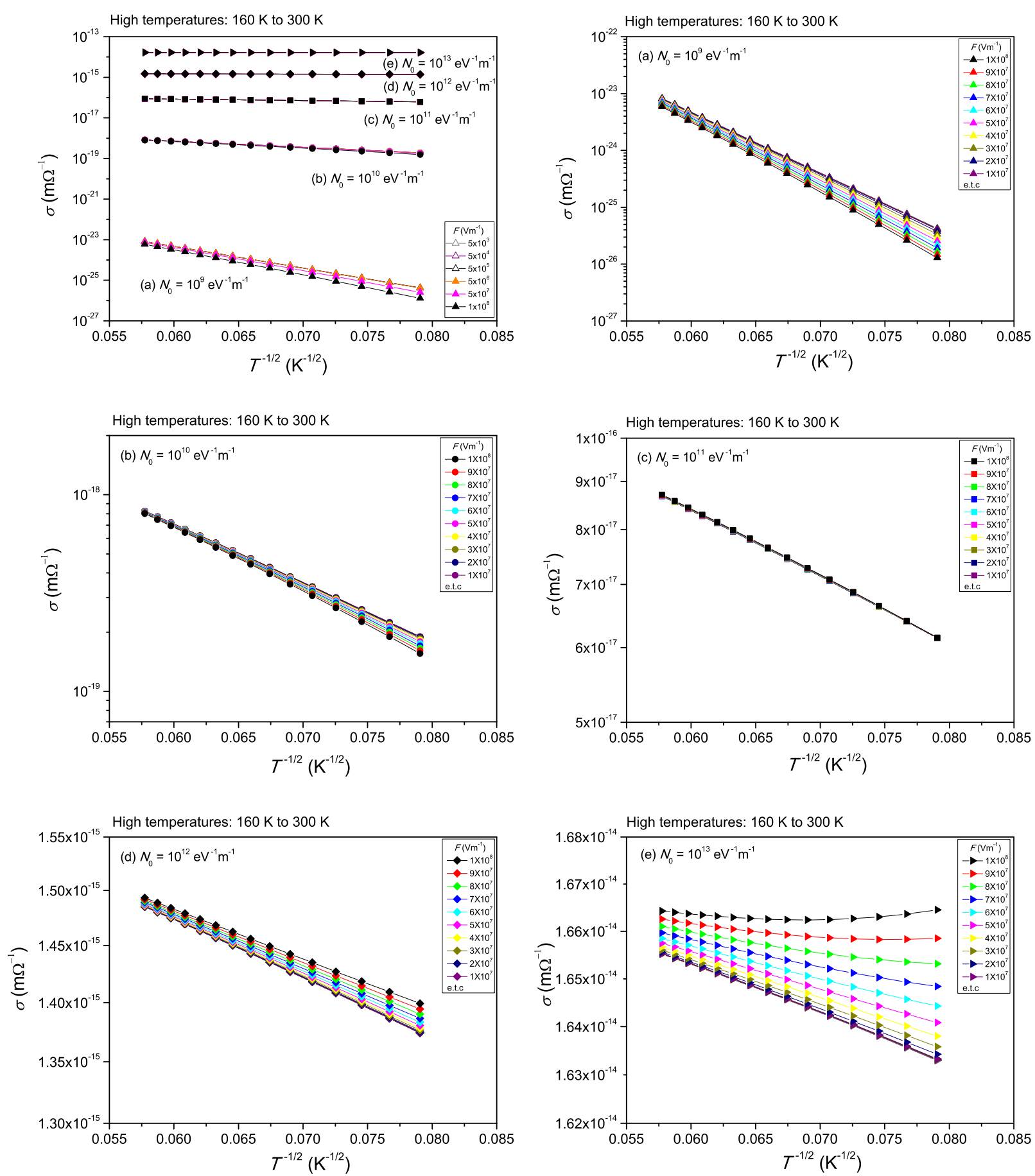

Figure 3. $\sigma$ versus $T^{-1 / 2}$, varying the density of states i.e. for $N_{0}=10^{9}-10^{13}$ $\mathrm{eV}^{-1} \mathrm{~m}^{-1}$ [cases (a) to (e), respectively]. $\alpha^{-1}=2 \AA . \quad T=(160-300) \mathrm{K}$ and $F=\left(5 \times 10^{3}-1 \times 10^{8}\right) \mathrm{Vm}^{-1}$.

field strengths. Finally, we observe that the temperature has a greater effect on the conductivity, the smaller the density of states is. Another aspect of the behaviour of the conductivity for different DOS is shown in Figure 4. $N_{0}=10^{9}-10^{13} \mathrm{eV}^{-1} \mathrm{~m}^{-1}$, 


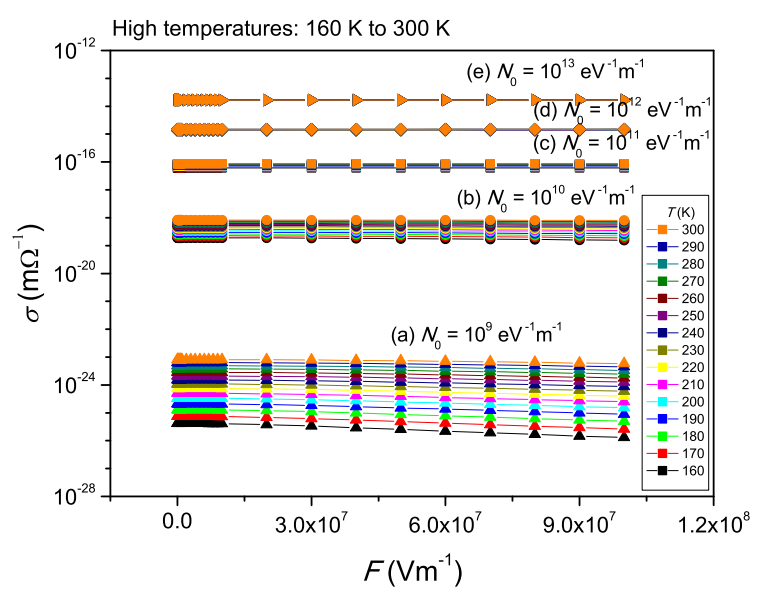

Figure 4. $\sigma$ versus $F$, varying the density of states i.e. for $N_{0}=10^{9}-10^{13} \mathrm{eV}^{-1} \mathrm{~m}^{-1}$ [cases (a) to (e), respectively]. $\alpha^{-1}=2 \AA . T=(160-300) \mathrm{K}$ and $F=\left(5 \times 10^{3}\right.$ $\left.1 \times 10^{8}\right) \mathrm{Vm}^{-1}$.

$T=(160-300) \mathrm{K}$ and $F=\left(5 \times 10^{3}-1 \times 10^{8}\right) \mathrm{Vm}^{-1}$.

Let us denote by $\sigma_{0}$ the ohmic value of the conductivity, i.e. $\sigma_{0}=\lim _{F \rightarrow 0}(\sigma)$. In order to show the deviation of $\sigma$ from $\sigma_{0}$ under the influence of both $F$ and $T$ we present Fig. 5 which shows $\sigma / \sigma_{0}$ versus $\beta=e F / 2 \alpha k_{B} T$ for (a) $N_{0}=10^{9} \mathrm{eV}^{-1} \mathrm{~m}^{-1}$ and (c) $N_{0}=10^{11} \mathrm{eV}^{-1} \mathrm{~m}^{-1}$, respectively. $F=\left(5 \times 10^{3}-1 \times 10^{8}\right) \mathrm{Vm}^{-1}$ and $T=(160-300)$ K. We observe that the effect of the external stimuli $F$ and $T$ on $\sigma$ depends strongly on the value of the density of states that characterizes the system. For (a) $N_{0}=10^{9}$ $\mathrm{eV}^{-1} \mathrm{~m}^{-1}$ the conductivity decreases from its ohmic value in the specific range of $F$ and $T$, while for (c) $N_{0}=10^{11} \mathrm{eV}^{-1} \mathrm{~m}^{-1}$ the conductivity generally increases and it is higher for higher temperatures. We notice that the variation of $\sigma / \sigma_{0}$ versus $\beta=e F / 2 \alpha k_{B} T$ is generally small especially in contrast to the corresponding variation at low temperatures studied later on Subsection 3.2.

Figure 6 shows the conductivity for different values of the spatial extent of the localized electronic wave function, i.e. for $\alpha^{-1}=(1-5) \AA$. Here we have chosen case (c) $N_{0}=10^{11} \mathrm{eV}^{-1} \mathrm{~m}^{-1}$ for the density of states. $T=(160-300) \mathrm{K}$ and $F=\left(5 \times 10^{3}\right.$ $\left.1 \times 10^{8}\right) \mathrm{Vm}^{-1}$. We observe that smaller $\alpha^{-1}$ (more localized carriers) leads to smaller $\sigma$. Particularly, five times increase of $\alpha^{-1}$ leads to two orders of magnitude greater conductivity. In addition, in Fig. 7 we observe that $\frac{d \sigma}{d \beta}>0$ for any temperature when $\alpha^{-1}=3,4$ and $5 \stackrel{\circ}{A}$, while $\frac{d \sigma}{d \beta}<0$ when $\alpha^{-1}=1 \stackrel{\circ}{A}$. For the case $\alpha^{-1}=2 \stackrel{\circ}{A}$ cf. Fig. 5 (II). In other words, the strength of the localization which determines the size of the formed polaron, along with the density of states which characterizes the system, are both two key factors for the conductivity and its dependance on $F$ and $T$. 

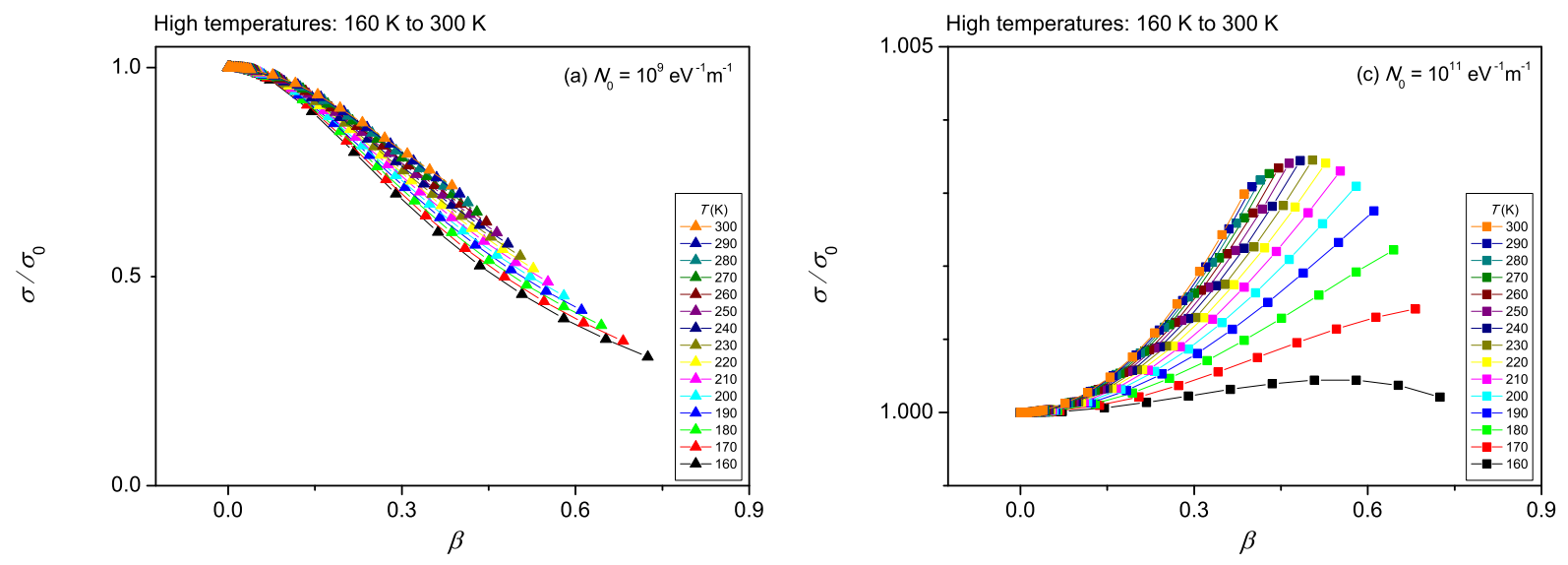

Figure 5. $\sigma / \sigma_{0}$ versus $\beta=e F / 2 \alpha k_{B} T$. (I) Left Panel. Case (a) $N_{0}=10^{9} \mathrm{eV}^{-1} \mathrm{~m}^{-1}$. (II) Right Panel. Case (c) $N_{0}=10^{11} \mathrm{eV}^{-1} \mathrm{~m}^{-1}$. In both panels $\alpha^{-1}=2 \AA, F=\left(5 \times 10^{3}\right.$ $\left.-1 \times 10^{8}\right) \mathrm{Vm}^{-1}$ and $T=(160-300) \mathrm{K}$.
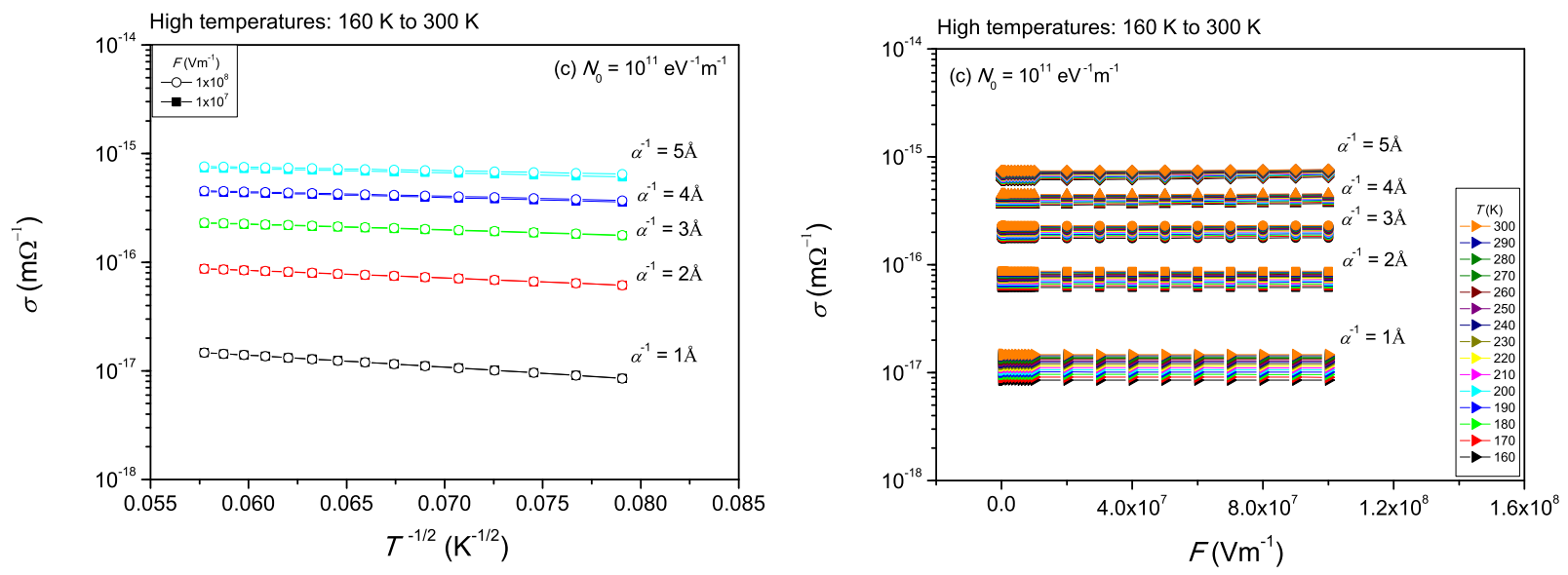

Figure 6. (I) Left Panel. $\sigma$ versus $T^{-1 / 2}$. (II) Right Panel. $\sigma$ versus $F$. The spatial extent of the localized electronic wave function is taken $\alpha^{-1}=(1-5) \AA$. Here we have chosen case (c) $N_{0}=10^{11} \mathrm{eV}^{-1} \mathrm{~m}^{-1}$ for the density of states. $T=(160-300) \mathrm{K}$ and $F=\left(5 \times 10^{3}-1 \times 10^{8}\right) \mathrm{Vm}^{-1}$.

\subsection{Low Temperatures}

For the temperature range $T=(10-150) \mathrm{K}$, Fig. 8 presents $\sigma$ as a function of $T^{-1 / 2}$ for different magnitudes of the DOS, i.e. $N_{0}=\left(10^{9}-10^{11}\right) \mathrm{eV}^{-1} \mathrm{~m}^{-1}$ [cases (a) to (c), respectively]. $\alpha^{-1}=2 \AA . F=\left(5 \times 10^{3}-1 \times 10^{8}\right) \mathrm{Vm}^{-1}$. Again, we depict our results as a function of $T^{-1 / 2}$ in order to compare them with the analytically obtained formula $\ln \sigma \propto T^{-1 / 2}$ which holds for low up to moderate electric fields and was previously obtained by two of us [26] following a different theoretical treatment and taking into 


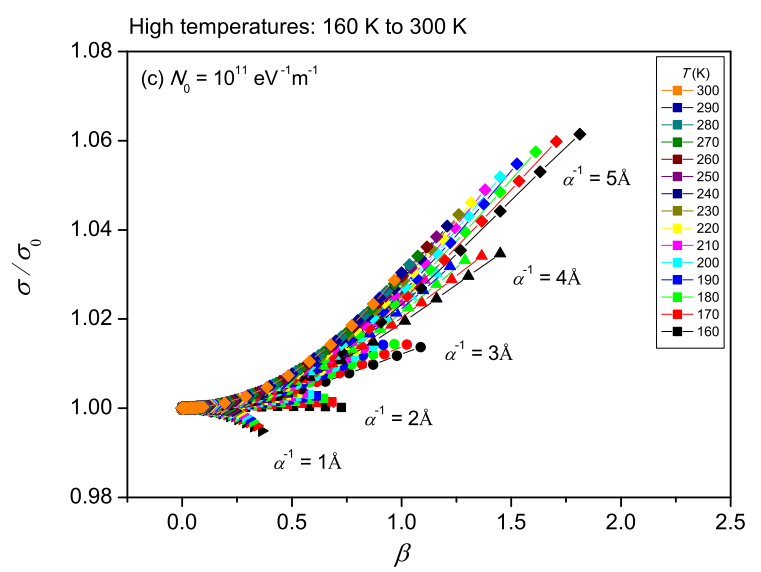

Figure 7. $\sigma / \sigma_{0}$ versus $\beta=e F / 2 \alpha k_{B} T$ for $\alpha^{-1}=(1-5) \AA$. For the density of states we have chosen (c) $N_{0}=10^{11} \mathrm{eV}^{-1} \mathrm{~m}^{-1} . T=(160-300) \mathrm{K}$ and $F=\left(5 \times 10^{3}-1 \times 10^{8}\right)$ $\mathrm{Vm}^{-1}$.

account the effect of correlations, namely

$$
\sigma^{l, c r}(F, T) \propto \exp \left[-\left(\frac{T_{0}^{l, c r}}{T}\right)^{1 / 2}\left(1-\frac{F^{2}}{g(T)}\right)^{1 / 2}\right] .
$$

Here $T_{0}^{l, c r}=1.96 \alpha / k_{B} N_{0}$. We observe that higher density of states leads to higher conductivity in such a way that $\frac{d \sigma}{d N_{0}}$ is smaller for higher densities of states. In total, in this specific example, augmenting DOS by two orders of magnitude increases the conductivity by approximately tens of orders of magnitude. From these results we realize that the $T^{-1 / 2}$-behaviour of $\sigma$ [26] holds for low up to moderate electric fields. For higher values of $F$ the conductivity deviates from the $T^{-1 / 2}$-behaviour as $T$ decreases and this deviation appears to be larger the stronger the electric field is. This deviation is due to the constructive energetic contribution of the electric field which leads to the increase of the number of available sites that can host the carrier, i.e. the range $\bar{\Re}_{n n}^{l}$ does not depend solely on $T$. As the temperature further decreases, for strong enough electric fields, the range $\bar{\Re}_{n n}^{l}$ depends exclusively on the applied electric field, as essentially all hops are downward in energy. As a result, the conductivity does not depend on the temperature. In other words, there is a transition from thermally-assisted to field-assisted hopping.

We remind the reader that in the high temperature range $T=(160-300) \mathrm{K}$ discussed in subsection 3.1, the electric field affects the range between two sites in the "hopping space" both for the absorption and the emission branch, leading also to the appearance of the destructive role of the electric field. In contrast, this effect does not appear here in the low temperature range $T=(10$ - 150) $\mathrm{K}$, because in the corresponding expression for the range between two sites in the "hopping space" the electric field affects only the finite area of the absorption branch. 


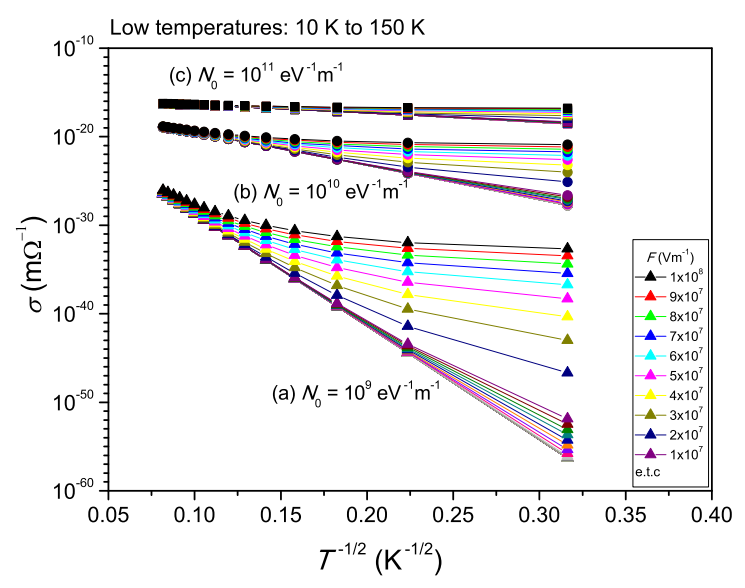

Figure 8. $\sigma$ versus $T^{-1 / 2}$ for cases (a) $N_{0}=10^{9} \mathrm{eV}^{-1} \mathrm{~m}^{-1}$, (b) $N_{0}=10^{10} \mathrm{eV}^{-1} \mathrm{~m}^{-1}$ and (c) $N_{0}=10^{11} \mathrm{eV}^{-1} \mathrm{~m}^{-1} \cdot \alpha^{-1}=2 \AA .9 . T=(10-150) \mathrm{K}$ and $F=\left(5 \times 10^{3}-1 \times 10^{8}\right)$ $\mathrm{Vm}^{-1}$.

Figure 9(I) presents the conductivity for different densities of states $N_{0}=10^{9}-10^{11}$ $\mathrm{eV}^{-1} \mathrm{~m}^{-1}$ as a function of the applied electric field. For low up to moderate electric fields the conductivity follows nicely the $F^{2}$-behaviour, as we expected from the analytical expression previously reported [24, 26]. Specifically, when the condition $F^{2} / g(T) \ll 1$ is satisfied, i.e. $e \alpha^{-1} F \ll 2 k_{B} T$, two of us have showed [26]

$$
\ln \sigma^{l, c r}(F, T) \propto \ln \sigma^{l, c r}(0, T)+h(F) / f^{l, c r}(T),
$$

where $\ln \sigma^{l, c r}(0, T)=-\left(T_{0}^{l, c r} / T\right)^{1 / 2}, h(F)=F^{2}$ and $f^{l, c r}(T)=\left[\left(T_{0}^{l, c r} / T\right)^{1 / 2} / 2 g(T)\right]^{-1}$. Increasing the electric field the conductivity becomes independent of the temperature and follows a $1 / F^{1 / 2}$-behaviour (Fig. 9(II)). The linear fit is of the form $y=A+B x$ with $A=-15.664 \pm 0.018, B=-11159.837 \pm 142.759$ and $R=-0.99959$. In the region between the $F^{2}$ and $1 / F^{1 / 2}$-behaviour, $\ln \sigma$ increases almost linearly with $F$.

The influence of both $F$ and $T$ on $\sigma$ is shown in Fig. 10, where we depict $\sigma / \sigma_{0}$ versus $\beta=e F / 2 \alpha k_{B} T$, for (a) $N_{0}=10^{9} \mathrm{eV}^{-1} \mathrm{~m}^{-1}$ and (c) $N_{0}=10^{11} \mathrm{eV}^{-1} \mathrm{~m}^{-1} . F=\left(5 \times 10^{3}\right.$ $\left.1 \times 10^{8}\right) \mathrm{Vm}^{-1}$ and $T=(10-150) \mathrm{K}$. The influence of $F$ on $\sigma$ depends on $T$, and it is greater at lower temperatures, while at higher temperatures the influence of $F$ decreases significantly. Comparing Fig. 10 with the corresponding one for high temperatures (cf. Fig. (5) we observe that the dependence of $\sigma / \sigma_{0}$ on $\beta=e F / 2 \alpha k_{B} T$ for low temperatures is very strong especially in contrast to the corresponding variation at high temperatures studied earlier on Subsection 3.1.

Fogler and Kelley [22], Raikh and Ruzin [25], and Ma et al. [23] refer also to the transition of a 1D disordered electron system from the ohmic to the non-ohmic behaviour, and in this respect their results are consistent with our results, for both low and high temperatures. For strong enough electric fields, Fogler and Kelley [22], Pollak and Riess [46], and Shklovskii [47], claim that in strong electric fields only the forward hops need to be considered, in contrast to Apsley and Hughes [40] who integrate over the entire space. Our methodology follows in that sense Apsley and Hughes [40] summing 

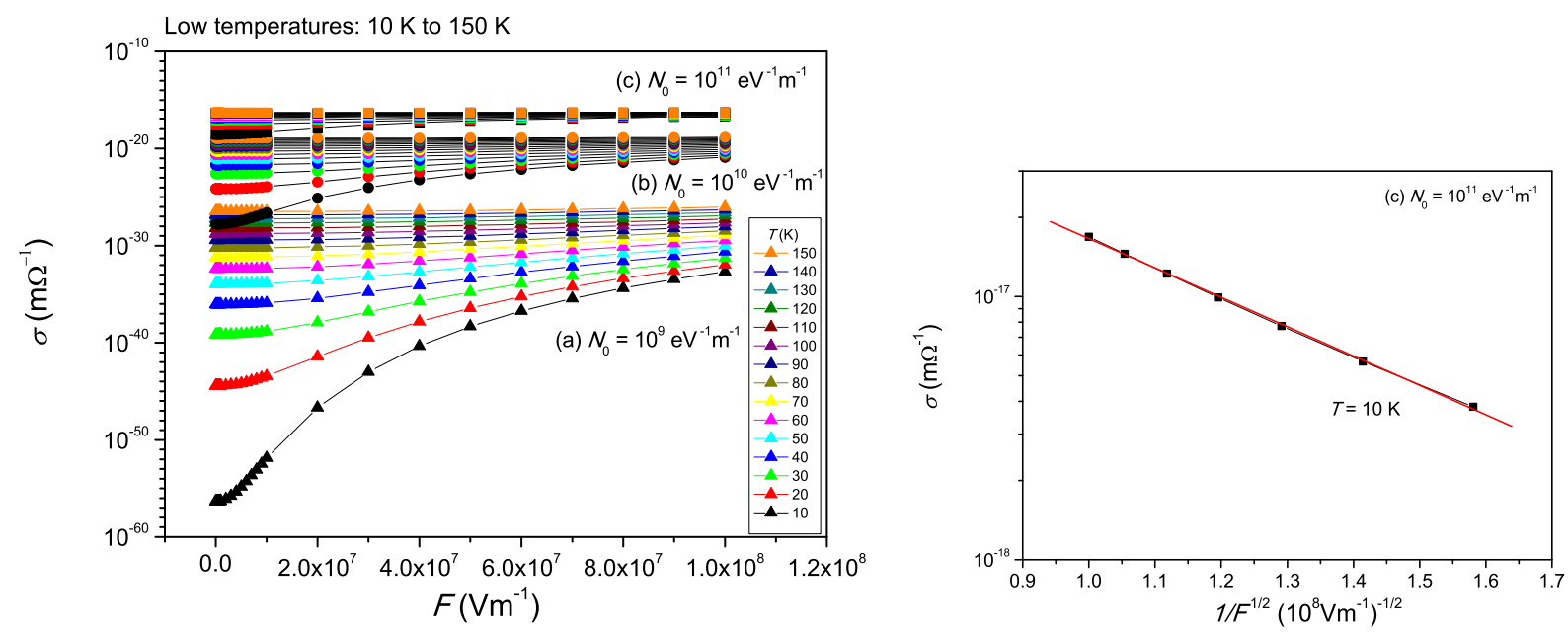

Figure 9. (I) Left Panel. $\sigma$ versus $F$ varying the density of states, i.e. for cases (a) $N_{0}=10^{9} \mathrm{eV}^{-1} \mathrm{~m}^{-1}$, (b) $N_{0}=10^{10} \mathrm{eV}^{-1} \mathrm{~m}^{-1}$ and (c) $N_{0}=10^{11} \mathrm{eV}^{-1} \mathrm{~m}^{-1}$. $F=\left(5 \times 10^{3}-1 \times 10^{8}\right) \mathrm{Vm}^{-1}$ and $T=(10-150) \mathrm{K}$. (II) Right Panel. $\sigma$ versus $F^{-1 / 2}$ for case (c) $N_{0}=10^{11} \mathrm{eV}^{-1} \mathrm{~m}^{-1}$ at $T=10 \mathrm{~K}$ and $F=\left(4 \times 10^{7}-1 \times 10^{8}\right) \mathrm{Vm}^{-1}$. In both panels $\alpha^{-1}=2 \stackrel{\AA}{A}$.
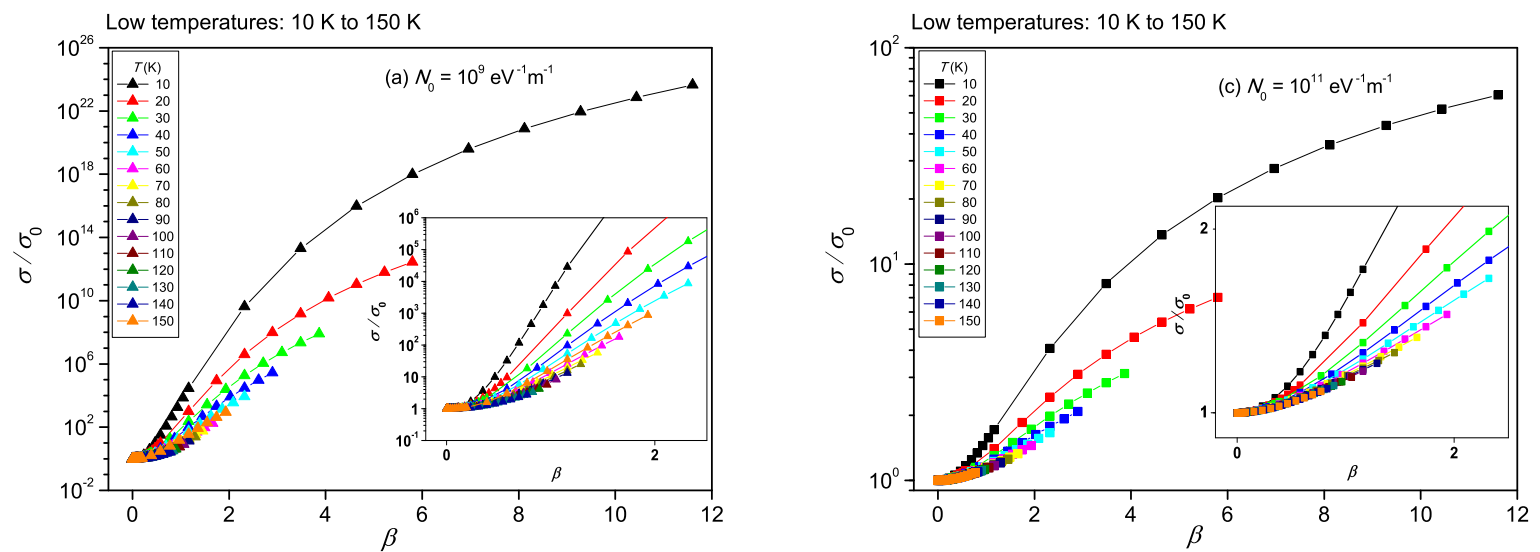

Figure 10. $\sigma / \sigma_{0}$ versus $\beta=e F / 2 \alpha k_{B} T$ for cases (a) $N_{0}=10^{9} \mathrm{eV}^{-1} \mathrm{~m}^{-1}$ and (c) $N_{0}=10^{11} \mathrm{eV}^{-1} \mathrm{~m}^{-1} \cdot \alpha^{-1}=2 \stackrel{A}{A} . F=\left(5 \times 10^{3}-1 \times 10^{8}\right) \mathrm{Vm}^{-1}$ and $T=(10-150)$ $\mathrm{K}$. The insets present enlargements of $\sigma / \sigma_{0}$ versus $e F / 2 \alpha k_{B} T$.

over forward as well as backward hops in our 1D polaron system. Bourbie et al. [48] study the $F$-dependence of the hopping conductivity in disordered $3 D$ electron systems. They propose -among other mechanisms- that $\sigma$ decreases with increasing $F$, when $F$ is strong enough to affect the tunneling probability. This is due to the influence of $F$ on the number of percolation paths, in the sense that increasing $F$ certain paths become disallowed. This is in analogy with our discussion about the destructive role of $F$ at high $F$ and low DOS. Bourbie et al. [29], taking into account that " $F$ affects the effective dimension of the transport path, reducing it in the high- $F$ regime to $1 D$ ", showed that 
when $F=10^{8} \mathrm{Vm}^{-1}, T \approx(200-330) \mathrm{K}, \alpha^{-1}=2 \AA$ and $N_{0}=10^{12} \mathrm{eV}^{-1} \mathrm{~m}^{-1}$, the conductivity decreases with increasing temperature. This has been attributed to the competition between thermal-assisted and field-assisted hopping. We have obtained a similar behaviour for $\sigma$ when $F=10^{8} \mathrm{Vm}^{-1}$, at high temperatures $T=(160-300) \mathrm{K}$,

$\alpha^{-1}=2 \AA$ and $N_{0}=10^{13} \mathrm{eV}^{-1} \mathrm{~m}^{-1}$ (cf. Fig. (3)). Bourbie et al. have also included different forms of the DOS and mention that these different DOS lead to very similar $F$-dependence of $\sigma$. D. Bourbie [30] also used some different values for the extent of the $3 D$ electronic wave function arriving at the result that greater extent of the carrier leads to higher conductivity in analogy with our results for $1 D$ polarons. However, we underline that all the above works [22, 40, 46, 47, 48, 29, 30] refer to electrons while we study polarons. Moreover, in our work we have scrutinized the importance of the magnitude of the density of states and the spatial extent of the localized electronic wave function (for arbitrary electric fields up to the polaron dissociation limit and for any "reasonable" temperature). Finally, in 1D systems the ionic or protonic transport might play a role in some cases $[49,50,51,52$. However, in the present manuscript we do not investigate such possibilities.

\section{Conclusion}

We showed that the strength of the localization which determines the size of the formed polaron along with the density of states are two key factors for the conductivity and its dependence on the electric field and the temperature either at high or at low temperatures. These aspects of small polaron hopping have been nearly ignored in the past.

To accomplish our task, we developed a novel theoretical approach inspired by the eminent work of Apsley and Hughes [40] in combination with the GMCM [13, 17, 12, 24, 26] and references therein. In addition, we combined analytical work with numerical calculations. In the present model the expression which determines the conductivity (cf. Eq. 34) depends on both the density of states and the extent of the electronic wave function. We varied the DOS by few orders of magnitude near values which are relevant to common 1D systems [27, 28, 29, 30] and the extent of the electronic wave function from 1 to $5 \AA$, i.e. for reasonable values for common organic molecules [31, 32]. Although in the present manuscript we used for simplicity a constant density of states, it is evident from Eq. 34 that one could also try an energy dependent DOS via the same approach. We examined $\sigma(T, F)$ for temperatures from 10 up to $300 \mathrm{~K}$ and up to the electric field values where polarons dissociate $\left(\approx 1 \times 10^{8} \mathrm{Vm}^{-1}\right)$.

We showed the the electric field plays both a constructive role by offering energy for the polaron hops and a destructive one, in the sense that the stronger it is the more it forces the polaron to jump opposite to the $\boldsymbol{F}$ direction prohibiting forward jumps to neighboring sites. The relative strength of these two roles depends on the DOS and localization regimes.

Our present method confirms that either for high temperatures or for low 
temperatures, higher density of states leads to higher conductivity. This is done in such a way that $\frac{d \sigma}{d N_{0}}$ is smaller for higher densities of states. Conclusively, augmenting DOS by few orders of magnitude increases the conductivity by many orders of magnitude.

For high temperatures, for the smaller densities of states $\frac{d \sigma}{d F}<0$ i.e. the conductivity is larger for lower $F$. This is due to the competitive role of the directionality imposed by the electric field and the temperature. This directionality affects destructively $\sigma$ when only few sites are available for the polaron i.e. for small DOS. We noticed that according to Eq. 16] and Fig. [(I), the electric field affects the range between two sites in the "hopping space" both for the absorption and the emission branch. On the contrary, this effect does not appear at low temperatures, because in the corresponding expression for the range between two sites in the "hopping space" at low temperatures (Eq. 25) and Fig. 2(I)), the electric field affects only the finite area of the absorption branch. We also noticed that the electric field plays a constructive role, too, due to its energy offer to the polarons. For "medium" DOS the available sites are numerous enough so that the directionality of $F$ hardly affects the conductivity. For higher DOS only the constructive energetic influence of the electric field appears. Now $\frac{d \sigma}{d F}>0$ i.e. the conductivity is larger for higher $F$. Finally, we observed that the temperature has a greater effect on the conductivity, the smaller the density of states is.

Our results confirmed that either for high or for low temperatures the behaviour $\ln \sigma \propto T^{-1 / 2}$ previously obtained by two of us [26], following a different theoretical treatment and taking into account the effect of correlations, holds for low up to moderate electric fields. Moreover, for low electric fields the conductivity follows the $F^{2}$-behaviour [24, 26], and increasing $F$ the conductivity becomes independent of $T$ and it follows a $1 / F^{1 / 2}$-behaviour while in the region between the $F^{2}$ and $1 / F^{1 / 2}$-behaviour, $\ln \sigma$ increases almost linearly with $F$.

We examined the deviation of conductivity from its ohmic value under the influence of both the external stimuli $F$ and $T$ (introducing $\beta=e F / 2 \alpha k_{B} T$ ). This was done either for high or for low temperatures, and for different DOS. We showed that $\sigma(\beta)$ depends strongly on the value of the DOS, and either decreasing or increasing $\sigma(\beta)$ could be observed. We noticed that the variation of $\sigma / \sigma_{0}$ versus $\beta$ is generally very small in high temperatures compared to the corresponding variation at low temperatures.

Finally, we studied the conductivity for different values of the spatial extent of the localized electronic wave function in the range $\alpha^{-1}=(1-5) \AA$. Our results confirm that more localized polarons exhibit smaller conductivity. Particularly, five times increase of $\alpha^{-1}$ lead to two orders of magnitude greater conductivity. Moreover, we showed that $\frac{d \sigma}{d \beta}>0$ for any $T$ when $\alpha^{-1}=3,4$ and $5 \AA$, while $\frac{d \sigma}{d \beta}<0$ when $\alpha^{-1}=1 \AA$. For the case $\alpha^{-1}=2 \AA$ we observed an intermediate behaviour.

In summary, we proved that the size of the polaron and the density of states are crucial factors for the behaviour of the conductivity and its dependence on the electric field and the temperature either at high or at low temperatures.

Acknowledgments C. S. acknowledges ELKE (National and Kapodistrian 
Density of states and extent of wave function ...

University of Athens) for financial support.

\section{Appendix}

The integrals $I_{1}$ and $I_{2}$, relevant at high temperatures, are given below:

$$
\begin{aligned}
& I_{1}=\sum_{0, \pi} \int_{\frac{1}{3}\left(E_{i}^{\prime}-\beta \bar{\Re}_{n n}^{h} \cos \theta\right)}^{\frac{1}{3}\left(2 \bar{\Re}_{n n}^{h}+E_{i}^{\prime}\right)} N\left(E_{j}^{\prime}\right)\left[1-f\left(E_{j}^{\prime}\right)\right]\left(\frac{\bar{\Re}_{n n}^{h}-\frac{3}{2} E_{j}^{\prime}+\frac{1}{2} E_{i}^{\prime}}{1+\frac{\beta}{2} \cos \theta}\right) \cos \theta d E_{j}^{\prime} . \\
& I_{2}=\sum_{0, \pi} \int_{\frac{1}{3}\left(E_{i}^{\prime}-\beta \bar{\Re}_{n n}^{h} \cos \theta\right)}^{\frac{1}{3}\left(2 \bar{\Re}_{n n}^{h}+E_{i}^{\prime}\right)} N\left(E_{j}^{\prime}\right)\left[1-f\left(E_{j}^{\prime}\right)\right] d E_{j}^{\prime} .
\end{aligned}
$$

As the integrals $I_{1}$ and $I_{2}$ diverge at $\beta=-2 / \cos \theta$, we change variables and integrate over $R^{\prime}$. Hence:

$$
\begin{aligned}
& I_{1}=\sum_{0, \pi} \int_{0}^{\bar{\Re}_{n n}^{h}} N\left(\frac{2}{3}\left[\bar{\Re}_{n n}^{h}-R^{\prime}\left(1+\frac{\beta}{2} \cos \theta\right)+\frac{E_{i}^{\prime}}{2}\right]\right) \times \\
& {\left[1-f\left(\frac{2}{3}\left[\bar{\Re}_{n n}^{h}-R^{\prime}\left(1+\frac{\beta}{2} \cos \theta\right)+\frac{E_{i}^{\prime}}{2}\right]\right)\right] R^{\prime} \cos \theta d R^{\prime} . } \\
& I_{2}=\sum_{0, \pi} \int_{0}^{\bar{\Re}_{n n}^{h}} N\left(\frac{2}{3}\left[\bar{\Re}_{n n}^{h}-R^{\prime}\left(1+\frac{\beta}{2} \cos \theta\right)+\frac{E_{i}^{\prime}}{2}\right]\right) \times \\
& {\left[1-f\left(\frac{2}{3}\left[\bar{\Re}_{n n}^{h}-R^{\prime}\left(1+\frac{\beta}{2} \cos \theta\right)+\frac{E_{i}^{\prime}}{2}\right]\right)\right] d R^{\prime} . }
\end{aligned}
$$

The integrals $I_{1}, I_{2}, I_{3}, I_{4}$ relevant at low temperatures are given below.

$$
\begin{aligned}
& I_{1}=\sum_{0, \pi} \int_{E_{i}^{*}-\beta \bar{\Re}_{n n}^{l} \cos \theta}^{E_{i}^{*}+\bar{\Re}_{n n}^{l}} N\left(E_{j}^{*}\right)\left[1-f\left(E_{j}^{*}\right)\right]\left(\frac{\bar{\Re}_{n n}^{l}-E_{j}^{*}+E_{i}^{*}}{1+\beta \cos \theta}\right) \cos \theta d E_{j}^{*} . \\
& I_{2}=\sum_{0, \pi} \int_{-\infty}^{E_{i}^{*}-\beta \bar{\Re}_{n n}^{l} \cos \theta} N\left(E_{j}^{*}\right)\left[1-f\left(E_{j}^{*}\right)\right] \bar{\Re}_{n n}^{l} \cos \theta d E_{j}^{*} . \\
& I_{3}=\sum_{0, \pi} \int_{E_{i}^{*}-\beta \bar{\Re}_{n n}^{l} \cos \theta}^{E_{i}^{*}+\bar{\Re}_{n n}^{l}} N\left(E_{j}^{*}\right)\left[1-f\left(E_{j}^{*}\right)\right] d E_{j}^{*} \\
& I_{4}=\sum_{0, \pi} \int_{-\infty}^{E_{i}^{*}-\beta \bar{\Re}_{n n}^{l} \cos \theta} N\left(E_{j}^{*}\right)\left[1-f\left(E_{j}^{*}\right)\right] d E_{j}^{*} .
\end{aligned}
$$

As the integrals $I_{1}$ and $I_{3}$ diverge at $\beta=-2 / \cos \theta$, we change variables and integrate over $R^{\prime}$. Hence:

$$
\begin{aligned}
& \left.I_{1}=\sum_{0, \pi} \int_{0}^{\bar{\Re}_{n n}^{l}} N\left(\bar{\Re}_{n n}^{l}-R^{\prime}(1+\beta \cos \theta)+E_{i}^{*}\right)\right)\left[1-f\left(\bar{\Re}_{n n}^{l}-R^{\prime}(1+\beta \cos \theta)+E_{i}^{*}\right)\right] R^{\prime} \cos \theta d R^{\prime} . \\
& \left.I_{3}=\sum_{0, \pi} \int_{0}^{\bar{\Re}_{n n}^{l}} N\left(\bar{\Re}_{n n}^{l}-R^{\prime}(1+\beta \cos \theta)+E_{i}^{*}\right)\right)\left[1-f\left(\bar{\Re}_{n n}^{l}-R^{\prime}(1+\beta \cos \theta)+E_{i}^{*}\right)\right] d R^{\prime} .
\end{aligned}
$$




\section{References}

[1] Gleve B, Hartenstein B, Baranovskii S D, Scheidler M, Thomas P and Bössler H 1995 Phys. Rev. $B \mathbf{5 1} 16705$

[2] Nebel C E, Street R A, Johnson N M and Kocka J 1992 Phys. Rev. B 466789

[3] Godet C and Kumar S 2003 Phil. Mag. 833351

[4] Cumings J and Zettl A 2004 Phys. Rev. Lett. 93086801

[5] Tang Z K, Sun H D and Wang J 2000 Physica B 279200

[6] Campbell I H, Smith D L, Neef C J and Ferraris J P 1999 Appl. Phys. Lett. 742809

[7] Mozer A Z, Sariciftci N S, Pivrikas A, Österbacka R, Juska G, Brassat L and Bässler" H 2005 Phys. Rev. B $\mathbf{7 1} 035214$

[8] Novikov S V, Dunlap D H, Kenkre V M, Parris P E and Vannikov A V 1998 Phys. Rev. Lett. 81 4472

[9] Aleshin A N, Lee J Y, Chu S W, Lee S W, Kim B, Ahn S J and Park Y W 2004 Phys. Rev. B 69 214203

[10] Yu Z G, Smith D L, Saxena A, Martin R L and Bishop A R 2000 Phys. Rev. Lett. 84721

[11] Yu Z G, Smith D L, Saxena A, Martin R L and Bishop A R 2001 Phys. Rev. B 63085202

[12] Triberis G. P. and Dimakogianni M 2009 Rec. Pat. Nanotechnol. 3135

[13] Triberis G P, Simserides C and Karavolas V C 2005 J. Phys.: Condens. Matter 172681

[14] Triberis G P and Friedman L R 1981 J. Phys. C.: Solid State Phys. 144631

[15] Tran P, Alavi B and Gruner G 2000 Phys. Rev. Lett. 851564

[16] Yoo K-H, Ha D H, Lee J-O, Park J W, Kim J, Kim J J, Lee H-Y, Kawai T and Choi H-Y 2001 Phys. Rev. Lett. 87198102

[17] Triberis G P and Dimakogianni M 2009 J. Phys.: Condens. Matter 21035114

[18] Inomata A, Shimomura T, Heike S, Fujimori M, Hashizume T and Ito K 2006 J. Phys. Soc. Japan 75074803

[19] Schuster G B 2000 Acc. Chem. Res. 33253

[20] Carell T, Behrens C and Gierlich J 2003 J. Org. Biomol. Chem. 12221

[21] Takada T, Kawai K, Fujitsuka M and Majima T 2004 Natl. Acad. Sci. (USA) 10114002

[22] Fogler M M and Kelley R S 2005 Phys. Rev. Lett. 95166604

[23] Ma S, Xu H, Li Y and Song Z 2007 Physica B 39855

[24] Triberis G P and Dimakogianni M 2009 J. Phys.: Condens. Matter 21385406

[25] Raikh M E and Ruzin I M 1989 Sov. Phys. JETP 68642

[26] Dimakogianni M and Triberis G P 2010 J. Phys.: Condens. Matter 22355305

[27] Hawke L G D, Kalosakas G and Simserides C 2010 Eur. Phys. J. E 32291

[28] Hawke L G D, Kalosakas G and Simserides C 2011 Eur. Phys. J. E 34118

[29] Bourbie D, Ikrelef N, Driss-Khodja K and Nedellec P 2007 Phys. Rev. B 75184204

[30] Bourbie D 2011 Appl. Phys. Lett. 98012104

[31] Hawke L G D, Kalosakas G and Simserides C 2009 Mol. Phys. 1071755

[32] Hawke L G D, Simserides C and Kalosakas G 2009 Mater. Sci. Eng. B 165266

[33] Triberis G P and Friedman L R 1986 J. Non-Crystalline Solids 7929

[34] Emin D 1975 Adv. Phys. 24305

[35] Triberis G P 1985 Phys. Stat. Sol. (b) 132641

[36] Kubo R 1957 J. Phys. Soc. Japan 121203

[37] Ambegaokar V, Halperin B I and Langer J S 1971 Phys. Rev. B 42612

[38] Emin D 1975 Phys. Rev. Lett. 35882

[39] Fehske H and Trugman S A 2007 Numerical Solution of the Holstein Polaron Problem p 396 in Polarons in Advanced Materials (ed Alexandrov A S) (Springer)

[40] Apsley N and Hughes H P 1975 Phil. Mag. 311327

[41] Triberis G P, Zianni X, Yannacopoulos A N and Karavolas V C 1991 J. Phys. Condens. Matter 3 337 
[42] Triberis G P 1992 Phil. Mag. 65631

[43] Rakhmanova S V and Conwell E M 1999 Appl. Phys. Lett. 751518

[44] Liu X, Gao K, Fu J, Li Y, Wei J and Xie S 2006 Phys. Rev. B 74172301

[45] Qiu Y and Zhu L-P 2009 J. Chem. Phys. 131134903

[46] Pollak M and Riess I 1976 J. Phys. C.: Solid State Phys. 92339

[47] Shklovskii B I 1973 Sov. Phys. Semicond. 61964

[48] Bourbie D, Ikrelef N and Nedellec P 2004 Phys. Stat. Sol. (c) 179

[49] Wang J 2008 Phys. Rev. B $\mathbf{7 8} 245304$

[50] Pavlenko N 2000 J. Chem. Phys. 1128637

[51] Pavlenko N 2003 J. Phys.: Condens. Matter 15291

[52] Rak J, Makowska J, Voityuk A A 2006 Chemical Physics 325567 